\title{
eJRIEPS
}

Ejournal de la recherche sur l'intervention en éducation physique et sport

$14 \mid 2008$

Varia

\section{Analyse des pratiques d'enseignement du judo en EPS}

Denis Loizon, Éric Margnes et André Terrisse

\section{(2) OpenEdition}

\section{Journals}

Édition électronique

URL : https://journals.openedition.org/ejrieps/5911

DOI : 10.4000/ejrieps.5911

ISSN : 2105-0821

Éditeur

ELLIADD

\section{Référence électronique}

Denis Loizon, Éric Margnes et André Terrisse, « Analyse des pratiques d'enseignement du judo en EPS », eJRIEPS [En ligne], 14 | 2008, mis en ligne le 01 avril 2008, consulté le 17 juin 2021. URL : http:// journals.openedition.org/ejrieps/5911; DOI : https://doi.org/10.4000/ejrieps.5911

\section{(0) $(1)$}

La revue eJRIEPS est mise à disposition selon les termes de la Creative Commons Attribution 4.0 International License. 


\section{Analyse des pratiques d'enseignement du judo en EPS}

Denis Loizon*, Eric Margnes** \& André Terrisse***

* IUFM de Bourgogne. Équipe AP3E et LEMME (EA 3042), Université Paul Sabatier, Toulouse

**Université de Pau et des Pays de l'Adour - Département STAPS de Tarbes (LAPS) Chercheur associé LEMME (E.A. 3042 - Equipe AP3E) - Toulouse

***IUFM Midi-Pyrénées - Équipe AP3E, LEMME (EA 3042), Université Paul Sabatier, Toulouse

\section{Résumé}

Cette recherche en didactique de nature descriptive a pour but d'analyser les pratiques effectives des enseignants d'EPS, afin de rendre compte des différents objets de savoir qui sont réellement enseignés par les professeurs d'EPS, qui utilisent l'activité judo comme moyen d'enseignement.

L'identification de certaines variables didactiques nous a permis de mettre en évidence l'enseignement de savoirs technique et stratégique ainsi que leur complémentarité. Grâce à l'analyse des pratiques effectives, nous avons également identifié d'autres objets de savoirs qui sont transmis par ces professeurs d'EPS comme les savoirs sécuritaire, réglementaire, culturel ou éthique. L'utilisation d'entretiens post-leçon a confirmé ces intentions didactiques pas toujours prévues dans les déclarations initiales des enseignants, confirmant en cela le décalage entre les intentions initiales et les prises de décision dans l'action. Mais ce qui fait l'originalité de notre travail, c'est avant tout la prise en compte du sujet et de son histoire personnelle, qui influence certaines prises de décisions didactiques.

À partir d'extraits issus de trois de nos quatre études de cas, nous montrerons le rôle de l'expérience personnelle de judoka, qui agit en arrière-plan du fonctionnement didactique ; c'est elle qui permet une « repersonnalisation » des objets de savoir enseignés.

Cet article reprend une partie de notre travail de thèse consacré à la recherche des objets de savoir transmis par les enseignants dans deux institutions différentes, le club et l'EPS (Loizon, 2004). Après avoir précisé le cadre général et les concepts centraux, nous 
présenterons les différentes étapes de cette recherche et la phase d'analyse des pratiques consacrée à l'enseignement du judo dans le cadre des cours d'EPS.

Cette recherche, qui prend les pratiques d'enseignement comme objet d'analyse, s'inscrit dans les axes de recherche ${ }^{1}$ développés au LEMME en didactique des APS $^{2}$ au sein d'un groupe qui tente de comprendre ce qui s'enseigne dans les sports de combat. La pratique que nous avons l'intention d'analyser est celle des professeurs d'EPS, qui prennent le judo comme moyen d'enseignement. Le but de cette recherche est de mieux comprendre ces pratiques dans une visée heuristique (Bru, 2002), puisqu'il s'agit ici de rendre compte de la façon dont le judo est enseigné dans le milieu scolaire. Nous souhaitons analyser plus particulièrement ce qui est mis en œuvre par le professionnel pour transmettre le ou les savoirs du judo dans la leçon d'EPS.

\section{Cadre théorique}

Notre recherche s'inscrit dans le cadre général des études transpositives qui s'intéressent aux transformations que subit le savoir avant d'être appris par l'élève. Le professeur d'EPS organise son enseignement à partir d'un traitement didactique de l'activité physique. L'étude de ce traitement didactique est appelée transposition didactique, pour reprendre l'expression de Verret (1975) à l'origine de ce concept. Celui-ci est devenu un outil théorique indispensable (Terrisse, Léziart, 1997), dans la mesure où il permet de comprendre les différentes transformations que subit le savoir depuis son origine jusqu'à son acquisition par l'élève. Quel est donc le savoir enseigné dans ce cadre scolaire ? Quelles sont les transformations que subit ce savoir ? Comment peut-on expliquer ces transformations? Autant de questions qui vont devenir centrales dans cette analyse des pratiques d'enseignement.

\section{1. Contexte théorique}

Pour mieux comprendre cette première étape de la transposition didactique, nous utilisons comme champ de référence du savoir ce que Martinand (1986) appelle "les pratiques sociales de référence " dans le sens où l'activité physique et sportive est porteuse d'un savoir de référence. Dans ce cas précis, il nous semble plus pertinent de parler de référence experte plutôt que référence savante. II est alors possible de retrouver cette référence experte dans les documents réalisés par la fédération française de judo (FFJ).

\footnotetext{
${ }^{1}$ Les axes de recherche sont les suivants : modélisation du savoir et transposition didactique, conceptions des apprenants et rapport au savoir, interactions didactiques.

${ }^{2}$ Activités Physiques et Sportives.
} 
Cette mise en texte du savoir expert est visible dans les deux méthodes d'enseignement réalisées par la FFJ, l'une en 1967, et l'autre plus récente en 1990, avec le concours des plus hauts gradés. Deux savoirs spécifiques au judo sont ainsi présentés dans ces deux méthodes: d'un côté le savoir technique et de l'autre le savoir stratégique; ils représentent ce que Terrisse (2000) appelle le savoir pour la pratique. Ces deux objets de savoirs relèvent selon nous, des savoirs fondamentaux qui caractérisent cette pratique sportive.

Le savoir technique peut être défini comme l'ensemble des techniques qui relèvent de l'activité judo, techniques codifiées au fil du temps par les experts ; il correspond dans la méthode de 1967 (FFJDA, 1967) à une description formelle essentiellement temporelle (le déséquilibre, le placement du corps de l'attaquant et la projection). Ce savoir technique composé des techniques de projection (judo debout) et des techniques de contrôle au sol (immobilisations, étranglements et clés de bras) constitue la base du judo permettant de reconnaître et d'identifier ce sport parmi les différents sports de combat (Janicot, Pouillart, 1997). Pour ces experts, cette base technique représente « l'invariant technique » qui se retrouve, quels que soient les pays ou les professeurs.

Le savoir stratégique peut être assimilé quant à lui, à un savoir procédural dans la mesure où il donne lieu à des énoncés de type « opératif » (Barbier, 1996). Nous pouvons alors concevoir ce savoir stratégique selon trois niveaux d'expression (Vergnaud, 1994) qui vont du niveau le plus personnel (savoir-faire personnel) au niveau le plus généralisable :

* Le niveau technico-tactique qui se concrétise par le système d'attaque et de défense. Ce système d'attaque très personnel organisé autour d'une technique préférentielle (le spécial) permet au judoka d'envisager différentes solutions face aux problèmes (actions ou réactions) posés par l'adversaire (Loizon, 2000).

* Le niveau des règles d'action car les énoncés possèdent un certain degré de généralisation (Gréhaigne et coll., 1993).

* Le troisième niveau correspond à l'énoncé de principes décrits sous des formes particulières qui vont des principes tactiques (Janicot, Pouillart, 1997) aux principes opérationnels (Cadière, Trilles, 1998).

1. 2. Les concepts centraux de la recherche

Le concept central de notre travail est le concept de variable didactique. C'est en utilisant ce concept de la didactique des mathématiques (Brousseau, 1998) que nous avons tenté de mettre en évidence les objets de savoir enseignés. Pour cela, nous avons avancé la 
thèse suivante: le savoir enseigné peut être identifié en analysant les variables didactiques utilisées dans les situations d'apprentissage. En effet, nous pensons avec Brousseau (1998) que la situation d'apprentissage conçue par le professeur, contient un ou des objets de savoir repérables au travers des consignes qui définissent cette situation; ces consignes agissent comme un ensemble de contraintes à l'intérieur desquelles l'élève est libre d'exercer sa rationalité (Margolinas, 1993). Ces contraintes caractérisent les variables didactiques qui fournissent le cadre dans lequel l'élève va travailler à la recherche des solutions d'un problème (Brousseau, 1986). L'enseignant peut alors jouer sur les consignes, c'est-à-dire les valeurs de ces variables, pour favoriser un nouvel apprentissage (Astolfi, Develay, 1989 ; Brousseau, 1986 ; Laborde, Vergnaud, 1994).

Nous proposons une définition de ce concept: "les variables didactiques sont des éléments constitutifs des situations d'apprentissage identifiables sous formes de contraintes sur lesquelles l'enseignant peut agir en fixant les valeurs afin de conduire l'élève vers un apprentissage donné " (Loizon, 2004). Chaque situation d'apprentissage est donc porteuse d'un ou plusieurs objets de savoir que l'on peut identifier à partir des différentes variables didactiques présentes dans la situation sous forme de consignes données par l'enseignant. Notre analyse des pratiques d'enseignement du judo en EPS va donc porter sur les situations d'apprentissage mises en œuvre par les professeurs d'EPS, et plus particulièrement sur les variables didactiques repérables dans ces situations à partir du discours de l'enseignant. Quelles sont alors les variables didactiques qu'il est possible d'associer aux deux savoirs spécifiques du judo que nous avons définis précédemment?

Une revue des ouvrages consacrés au judo ${ }^{3}$ nous a permis de mettre en évidence le savoir technique que chaque judoka doit démontrer à l'occasion des passages de grades. On y retrouve pour chacune des techniques, la présentation de la technique proprement dite ou forme de corps, associée à la description d'un placement ou déplacement de l'adversaire (uke) ainsi que le placement de celui qui attaque (tori). Ce savoir que nous avons qualifié de technique peut donc être associé à trois variables particulières qui sont communément admises dans l'enseignement traditionnel du judo, c'est-à-dire :

* Le Déplacement-placement de l'adversaire ;

* Le Tsukuri ou placement-déplacement de l'attaquant ;

${ }^{3}$ Cette revue de la littérature judo comprenait une quarantaine d'ouvrages et une vingtaine d'articles issus de la revue EPS. 
* La Technique proprement dite avec son nom codifié.

Pour identifier le savoir stratégique, nous avons également recherché ce qui pouvait prendre le statut de variable didactique dans cette littérature très spécifique car ce concept n'est pas toujours explicité ; on le retrouve sous des appellations très diverses : variables, variantes, évolutions, prolongements, variables de différenciation. Ce sont majoritairement les ouvrages ou les articles rédigés par des professeurs d'EPS qui nous ont permis de catégoriser (Bardin, 1998) les variables associées au savoir stratégique en trois grandes catégories $^{4}$ :

* La posture (latéralité, saisie, position).

* Les actions (attaque, défense, direction, signal).

* Le rapport d'opposition (tonicité, vitesse, score).

Le professeur peut donc aménager l'opposition à l'aide de ces trois catégories de variables comme le préconise Terrisse (1991) pour déséquilibrer le rapport de force à des fins d'apprentissage. Nous appelons cet ensemble de variables, les variables stratégiques car elles sont spécifiques de l'activité judo, à la différence d'autres variables que l'on peut rencontrer dans d'autres APS. Elles caractérisent ce que nous avons appelé le savoir stratégique par opposition au savoir technique. En effet, ces variables permettent à l'enseignant de construire des situations d'enseignement plus ou moins saturées en incertitude afin de faire apprendre aux élèves autre chose qu'un savoir technique formel et décontextualisé. L'ensemble des variables stratégiques et techniques constitue donc les variables spécifiques de l'activité judo.

Pour identifier les objets de savoir enseignés aux élèves, nous avons donc utilisé une grille d'analyse composée de ces trois variables techniques et de ces trois variables stratégiques avec des critères différenciateurs pour chacune des variables. Cette grille a également été utilisée pour analyser le savoir de référence dans la première étape de nos travaux ainsi que dans la seconde étape à propos des pratiques déclarées.

1. 3. Les étapes de la recherche

\section{3. 1. Le savoir de référence}

Dans le premier temps de notre phase exploratoire, nous nous sommes d'abord intéressés à ce qui pouvait constituer le savoir de référence pour les professeurs de judo qui enseignent en club. II s'agissait d'envisager le point de départ de la transposition

\footnotetext{
${ }^{4}$ Dans le cadre de cet article, nous ne détaillerons pas chacune de ces variables, ni les critères différenciateurs qui leur sont associés.
} 
didactique. Cette question du savoir de référence dans le cadre d'une activité physique et sportive comme le judo, a donc été abordée sous l'angle du savoir des experts (Johsua, 1996) à travers les différentes publications qui concernent l'enseignement du judo, avec une centration particulière sur les savoirs technique et stratégique. Pour évaluer le « poids » du savoir technique par rapport au savoir stratégique, nous avons analysé différents documents spécifiques au judo, soit 43 ouvrages qui portent tous uniquement sur le judo. Tous ces livres ont été écrits par des personnes considérées comme des experts de l'activité judo. Deux questions ont présidé à l'analyse de ces ouvrages : quel est le public visé par l'auteur? Quelles sont les variables spécifiques présentes dans le document?

Concernant la première question, nous pensions que le public visé par l'auteur pouvait avoir des répercussions sur la nature des objets de savoir proposés dans l'ouvrage car certains livres s'adressent davantage à des professeurs de judo comme les ouvrages fédéraux, alors que d'autres sont plutôt rédigés pour un public très large qui va du néophyte au judoka ceinture noire. Pour cela, nous avons élaboré trois catégories de public visé : le "tout public", qui peut s'étendre du débutant jusqu'au pratiquant ceinture noire, la catégorie "professeurs de judo" avec les documents produits par la FFJ, et la catégorie "professeurs d'EPS". Différentes sources d'information nous ont permis de retrouver les destinataires, soit dans le texte, soit par l'appartenance de l'auteur à un milieu donné, soit par l'éditeur.

Pour repérer les différentes variables spécifiques présentes dans les documents, nous avons eu recours à des critères différenciateurs (Bardin, 1998) pour chacune de nos variables techniques ou stratégiques. Pour le recueil des données, nous avons utilisé un codage binaire (Van Der Maren, 1996) qui nous a permis de relever la présence ou à l'absence de chacune des variables dans les documents étudiés.

L'analyse de ce corpus de référence contrasté autour de ces deux savoirs a montré que le savoir technique était prédominant dans les ouvrages et qu'il faisait l'objet d'une présentation longue et minutieuse chez une majorité d'experts. Toutefois, nous avons observé une différence concernant les ouvrages destinés aux professeurs d'EPS qui présentent quant à eux, un savoir stratégique (80\%) plus important que le savoir technique (59\%). Ainsi, la mise en mots d'une activité physique comme le judo fait déjà apparaître une présentation du savoir différente selon les auteurs. Les ouvrages écrits par des professeurs d'EPS insistent davantage sur le savoir stratégique dans cette activité alors que les ouvrages fédéraux semblent accorder autant d'importance à chacun des deux savoirs stratégique et technique. 
1. 3. 2. Les pratiques déclarées.

Le second temps de la phase exploratoire a porté sur le recueil d'un certain nombre d'informations à l'aide d'un questionnaire (Loizon et coll., 2004). L'objectif était de rendre compte des "pratiques d'enseignement déclarées " par les professeurs de judo à partir d'une analyse des variables didactiques qu'ils déclaraient utiliser dans leur enseignement. Nous avons identifié les objets de savoir qu'ils déclaraient transmettre à leurs élèves, à travers le croisement d'un certain nombre de réponses à des questions relatives à leurs conceptions de l'enseignement du judo, à leurs références (textes officiels, lectures, vidéos), à leurs moyens de formation continue ainsi qu'aux variables utilisées en cours.

Les résultats font apparaître le rôle prépondérant des stages comme moyen de formation continue des enseignants de judo. Cette formation continue des professeurs de judo se fait essentiellement dans des stages sous forme de transmission orale à l'aide de nombreuses démonstrations réalisées par les experts de la Fédération Française de Judo. C'est à l'occasion de ces stages que s'effectue la transmission des savoirs techniques par les formateurs nationaux qui constituent la référence experte. La quête d'un nouveau savoir technique, dans ces stages de formation en judo, pourrait s'expliquer par la volonté chez les professeurs de judo de renouveler le savoir qui subit l'usure du temps. Enfin, les réponses au questionnaire nous ont permis de mettre en évidence un autre savoir qui n'avait pas retenu notre attention au départ de cette étude, ce que nous avons appelé le savoir éthique car il incorpore les différentes valeurs mises en exergue par le Code Moral du judo. Toujours dans ce questionnaire, les professeurs de judo déclarent à notre grande surprise que ce savoir éthique serait le savoir le plus important qu'ils veulent transmettre, bien avant le savoir technique et loin devant le savoir stratégique. II s'agit là d'intentions exprimées dans les réponses à un questionnaire, mais qu'en est-il dans la réalité ?

\section{3. 3. Les pratiques réelles}

Après cette première phase exploratoire qui portait sur des déclarations, nous sommes allés observer les "pratiques réelles d'enseignement » chez quatre professeurs de judo (Loizon, 2005) et chez quatre professeurs d'EPS pour identifier ce qu'ils enseignaient réellement. D'un point de vue didactique, analyser les pratiques effectives d'enseignement d'une activité physique ou d'une discipline, c'est s'intéresser à la question du savoir qui constitue l'enjeu de la relation entre un professeur et des élèves dans un enseignement ordinaire. C'est aussi tenter de comprendre ce qui se joue dans cette relation. Nous allons présenter maintenant la phase d'analyse des pratiques d'enseignement du judo en EPS. 


\section{Méthode}

Pour répondre à notre question de départ où nous cherchions à identifier les savoirs enseignés en judo, nous avons donc choisi d'observer les pratiques d'enseignement de quatre professeurs d'EPS qui utilisent l'activité judo comme moyen d'enseignement. II faut souligner le fait que dans notre Académie, l'enseignement du judo en milieu scolaire est surtout réalisé par des professeurs d'EPS qui ont une grande expérience du judo ; c'est ce que nous avions relevé dans une étude précédente sur la base de questionnaires donnés en formation continue (Loizon, Terrisse, 2002). Tous les professeurs d'EPS que nous avons observés étaient ceintures noires de judo. Au travers de ces quatre études de cas, nous avons mis en évidence certains savoirs effectivement transmis. Cette méthode clinique « du cas par cas » nous a permis de rendre compte de la position subjective de chacun des enseignants (Terrisse, 2000).

Nous avons recueilli des données au moyen d'enregistrements vidéo et d'entretiens postleçon. Ces entretiens ont été réalisés immédiatement après la leçon de judo pour comparer nos observations avec les déclarations des professeurs. Au cours de ces entretiens de type semi-directif, nous avons questionné les enseignants sur leurs préparations de leçon (le contenu des préparations), sur les objectifs des leçons (définition des objectifs, pourquoi ces objectifs, effets attendus), ainsi que sur les variables utilisées dans les situations d'apprentissage proposées dans les leçons observées (quelles variables, quelles évolutions). Nous avons ainsi enregistré seize leçons d'EPS (quatre leçons observées sur un cycle de huit pour chacun des professeurs) avec des élèves de collège et de lycée, chacune des leçons étant suivie d'un entretien. Pour compléter nos premières observations, nous avons dû procéder à des entretiens d'après-coup. Ces entretiens sont très importants, car ils deviennent un mode de validation complémentaire aux autres recueils de données (Terrisse, 2000).

\section{Résultats et discussion}

Nous illustrons nos résultats en reprenant des éléments du discours des professeurs d'EPS : professeur DC, professeur GL, professeur GA et le professeur JB. Ce discours provient soit des leçons observées $(L)$, soit des entretiens post-leçon (Ent), soit des entretiens d'après-coup $(A C)$.

Grâce aux variables didactiques utilisées par ces enseignants lors des situations présentées aux élèves, nous avons observé l'enseignement de savoirs techniques; ici chez le professeur DC (L): « il s'agit de retrouver des sensations de gainage, impulsion 
jambe " ou encore chez le professeur JB (L): "tu peux attaquer avec ta jambe en réalisant un fauchage ". Nous avons également identifié des savoirs stratégiques chez le professeur DC (L) «Là, je ne peux pas porter mon mouvement en attaque vers l'avant, mais je peux enchaîner en arrière » ou encore chez le professeur JB (L) : " celui qui travaille, il utilise la force de l'autre ». Mais nous avons également identifié d'autres objets de savoir qui sont intégrés dans l'enseignement par ces professeurs d’EPS.

Si les savoirs technique et stratégique sont bien identifiés dans les situations d'apprentissage, ils ne peuvent être mis en œuvre que si le savoir sécuritaire est bien maîtrisé par les élèves dans le cas de l'enseignement du judo debout avec des projections. Ce savoir sécuritaire est essentiellement identifié à partir du savoir chuter et du savoir-faire chuter dans les situations d'apprentissage, ici chez le professeur DC (L) : « ils ne sentent pas comment poser le partenaire sur le côté, ils font courir un risque à leur uke. Je veux arriver à un résultat qui ne mette plus uke en péril », mais aussi dans le travail technique comme chez le professeur GA $(\mathrm{L})$ : " je garde le dos plat, c'est très important pour ne pas se faire mal au dos ». Tout au long des leçons observées nous avons vu apparaître des informations concernant le règlement de l'activité judo. Celui-ci est adapté par les enseignants d'EPS pour le rendre accessible aux élèves. II ne fait pas l'objet d'un enseignement particulier ; il est intégré dans la leçon sous forme de remarques à partir des observations du professeur DC : "Je vous rappelle que vous n'avez pas le droit de sortir de la surface de combat » ou du professeur JB : " je vous rappelle que l'on doit enlever tous les bijoux ». Ce savoir réglementaire fait bien l'objet d'une transmission, souvent sous forme de rappel. Pour ce qui concerne le savoir éthique, celui-ci se résume surtout au respect de l'autre qui est rappelé à maintes reprises par ces enseignants d'EPS (professeur DC : " Vous n'oubliez pas de saluer votre partenaire pour lui montrer que vous les respectez »).

Par ailleurs, nous avons également observé que ces professeurs d'EPS utilisaient des manières de faire particulières pour transmettre ces différents objets de savoir. Systématiquement, toutes les situations étaient présentées à partir d'une démonstration réalisée soit par l'enseignant seul, soit par l'enseignant avec un élève, soit par deux élèves. La transmission de ces différents objets de savoir se faisait soit à partir de situations d'enseignement (savoirs enseignés), soit sous forme d'une transmission directe à toute la classe sous la forme d'une information ou d'une remarque (savoirs transmis), soit encore de manière très personnalisée à l'occasion d'interactions didactiques privilégiées avec certains élèves. 
3. 1. La notion de filtre ou de "déjà-là "

Progressivement, au fil de ces entretiens post-leçon et des entretiens menés dans l'aprèscoup, nous avons atteint ce que Portugais (1998) appelle les couches d'intention et d'intentionnalité et que nous avons traduit par les « filtres » de l'action didactique.

En effet, Carnus (2001) a montré dans ses travaux sur les professeurs d'EPS en gymnastique, l'existence d'un « déjà-là décisionnel » en amont de toute décision didactique ; elle le situe au carrefour de trois autres « déjà-là » (expérientiel, conceptuel et intentionnel) en précisant qu'il peut fournir à l'enseignant « des motifs et mobiles possibles à la prise de décision. Il exerce une influence constante et latente sur les démarches décisionnelles de l'enseignant d'EPS » (Carnus, 2003). Elle souligne également le fait que le processus décisionnel de l'enseignant « est un élément dynamique de la transformation des contenus à enseigner en contenus effectivement enseignés; en quelque sorte, il éclaire le passage de l'intention à la décision ». Ainsi, dans le quotidien du métier d'enseignant, celui-ci est amené à prendre des décisions, c'est-à-dire faire des choix en situation didactique en fonction d'un certain nombre de déterminants objectifs ou subjectifs. Ce processus décisionnel est "à la fois un phénomène intime et spécifique » (Carnus, 2003) ce qui justifie pleinement l'option méthodologique que nous avons prise : l'approche clinique car nous pensons que seul le sujet enseignant peut rendre compte de ses actes. En amont de ces décisions, existent des intentions conscientes et non conscientes, ces fameuses couches d'intention évoquées par Portugais (1998). L'intention est entendue ici comme un mobile, un but qui va orienter les choix de l'enseignant avant et pendant l'action ; didactique au sens ou cette intention concerne l'enseignement d'un ou de plusieurs objets de savoir. Ce ou ces mobiles fonctionnent alors comme un « filtre » très particulier dans la mesure où il transforme, sélectionne, oriente les savoirs à enseigner ; il agit alors comme une sorte de transformateur.

Ce filtre particulier est toujours présent, il agit en permanence en tant que représentation du monde, de l'autre, du réel (Blaquier, 2003) : il est déjà-là. C'est ce « déjà-là » évoqué par Carnus $(2001,2003)$ que nous avons rencontré chez nos quatre sujets enseignants. Nous pourrions alors le définir comme un ensemble très hétéroclite composé de savoirs divers, mais aussi de croyances, de valeurs et d'intentions. C'est quelque chose qui préexiste au sujet avant le moment observé et qui s'enrichit au fil de la vie, à travers les différentes expériences. Le « déjà-là » s'inscrit donc sur un axe chronologique car il englobe tout ce qui s'est passé avant le moment observé par le chercheur ; il concerne donc le sujet et son histoire. Chapelle (2004) nous rappelle que « le préconscient joue un 
rôle de "filtre " en refoulant dans l'inconscient les pulsions inacceptables pour la conscience ». Pour paraphraser Freud, nous définirons ce filtre, ce « déjà-là " comme une forme de surmoi (Freud, 2001) avec parfois des effets de censure car le " déjà-là » représente à tout jamais l'influence sur le sujet enseignant de son expérience personnelle de pratiquant comme nous le montrerons.

3. 2. Les différents " déjà-là »

Nous avons donc vu apparaître dans les discours des enseignants différents " déjà-là », différentes couches, que nous avons tenté de caractériser, et qui constituent pour nous autant de «filtres » par lesquels les savoirs se transforment progressivement. Dans les entretiens d'après-coup, nous avons compris que ces différents filtres, ces "déjà-là" qui préexistent à l'enseignement, se sont construits au fil des expériences qu'ont pu vivre les professeurs alors qu'ils étaient encore jeunes judokas. Nous avons alors constaté que les professeurs d'EPS mettaient en scène leur propre rapport au savoir dans les situations d'enseignement qu'ils proposaient à leurs élèves (Blanchard-Laville, 2001). Ceci nous a permis de souligner l'importance de l'expérience personnelle de pratiquant de judo qui agit en arrière plan du système didactique comme un filtre dans lequel les savoirs à enseigner seraient transformés, rejetés ou ajoutés.

C'est en relisant les entretiens post-leçon et les entretiens d'après-coup que nous avons pu reconstruire a posteriori, ces différents filtres qui viennent transformer les savoirs à enseigner en savoirs enseignés comme le constate Chevallard (1991) lorsqu'il décrit les mécanismes de la transposition didactique interne. Nous avons donc caractérisé quatre filtres, quatre " déjà-là », chez chaque enseignant en nous référent à Terrisse (1994). Le premier que nous qualifions de « déjà-là intentionnel spécifique » correspond aux intentions didactiques se rapportant à la leçon observée tandis que le second, le « déjà-là intentionnel général » prend en compte les intentions qui s'étendent sur une période plus longue comme le cycle en EPS. Nous avons donc distingué deux niveaux d'intentions: celles qui sont dans le court terme de la séance observée, et celles relative à une plus longue période. Nous avons eu accès à ces deux couches d'intentions didactiques dans les entretiens post-leçons quand nous avons questionné les professeurs au sujet à propos de leurs objectifs. Le troisième filtre, le « déjà-là conceptuel » renvoie aux conceptions de l'enseignant, c'est-à-dire aux éléments qui structurent en profondeur les intentions didactiques (Durand, 1998) comme les croyances et les valeurs de chaque enseignant. Certains de ces éléments sont apparus dans les entretiens post-leçon et d'autres dans les entretiens d'après-coup. Le dernier filtre, quant à lui, fait référence à l'expérience du 
professeur ; il s'agit du "déjà-là expérientiel ». Nous l'avons identifié très clairement à travers l'expérience relatée par les professeurs d'EPS. En reprenant la première topique de Freud, et pour mieux situer les registres dans lesquels se situent les déjà-là, nous nous risquerons à dire que les deux « déjà-là intentionnels » sont conscients ; il s'agit de buts clairement exprimés alors que le « déjà-là conceptuel » se trouverait en partie plutôt dans le préconscient. Le « déjà-là expérientiel » serait quant à lui, bien enfoui dans le préconscient pour ce qui concerne l'expérience de pratiquant. Ce registre du préconscient renvoie à ce que Vermersch appelle le pré-réfléchi quand il travaille sur l'explicitation de l'action (Vermersch, 1994).

Ces quatre "déjà-là » agissent donc comme des filtres personnels qui influencent fortement le choix des savoirs à enseigner (les intentions didactiques), mais aussi et surtout les décisions didactiques prises dans la leçon, c'est-à-dire les choix d'objets de savoirs à enseigner pendant la leçon. On peut alors comprendre l'effet de la transposition didactique interne : deux enseignants qui doivent enseigner les mêmes objets de savoirs n'enseigneront jamais réellement les mêmes objets de savoirs car ils ont des filtres, des « déjà-là » fondamentalement différents à cause de leur histoire personnelle.

En comparant ce que les professeurs nous avaient annoncé avoir enseigné dans les entretiens post-leçon, et notre analyse de ce qui avait été effectivement enseigné, nous avons observé de nombreux écarts qui nous ont conduit à demander des explications complémentaires dans les entretiens d'après-coup. C'est au cours de ces entretiens d'après-coup, d'abord semi-dirigés puis libres, que les enseignants nous ont raconté leur histoire personnelle de pratiquant de judo.

3. 3. Illustrations avec le cas du professeur DC

Nous allons illustrer nos propos en nous référent d'abord au cas du professeur DC en reprenant des extraits d'entretiens post-leçon (Ent) et d'entretiens après-coup (AC), puis nous complèterons ces illustrations à partir des autres cas, surtout pour montrer l'influence de l'expérience personnelle de pratiquant. La difficulté pour le chercheur, c'est bien d'arriver à faire le tri dans le flot d'informations qui fait suite à une question pourtant précise posée dans les entretiens.

Lors du premier entretien post-leçon, le professeur DC nous précise l'objectif de sa leçon : « Aujourd'hui, ça se situait sur le gainage qui fait partie d'un processus global de gainage des chevilles, des genoux, des épaules qui permettent d'évoluer dans l'espace en étant propulsé et non pas en tirant l'autre (Ent 1) ». Le travail technique ainsi proposé par le professeur DC ne concerne pas l'apprentissage de techniques proprement dites, mais il 
permet surtout d'acquérir une posture que le judoka doit garder pour être efficace. En évoquant la notion d'efficacité qu'il tente aussi de faire acquérir à ses élèves, le professeur définit celle-ci en relation avec l'intégrité physique du pratiquant et la gestion de la vie physique d'adulte: "Mais l'efficacité, je la place aussi dans la possibilité de faire encore du judo à quarante ans (...). Je travaille donc le gainage de toutes les articulations pour un gainage de tout le corps permettant un déplacement dans l'espace différent du tirerpousser habituel ; l'efficacité, c'est aussi faire du judo encore à 40 ans sans s'esquinter, ni les genoux, ni le dos (Ent 1)».

$\mathrm{Au}$ travers de ces quelques extraits d'entretien, nous constatons déjà des intentions didactiques légèrement différentes dans la mesure où elles se situent sur deux niveaux que nous distinguons : un « déjà-là intentionnel » présent avec les intentions spécifiques relatives à la leçon observée avec le travail technique de gainage du corps, et un « déjà-là intentionnel » plus général autour du thème de la sécurité dans la pratique. Dans ce même entretien, en une phrase, il nous livre ensuite trois valeurs qui constituent le socle de ses conceptions, son « déjà-là conceptuel »; nous pourrions les résumer ainsi : la technique au service de l'esthétique, de l'efficacité et du plaisir: "C'est le travail du geste, esthétique au sein de l'efficacité, c'est ça le plaisir (Ent 1) ». Le professeur reviendra à de nombreuses reprises au cours des entretiens sur ces notions très importantes pour lui : " Travailler la beauté du geste, ce n'est pas de l'esthétique pour de l'esthétique, c'est pour des sensations agréables de pratique agréable. Mais cela doit rester efficace (Ent 1). Parce que j'ai senti que cela les ramenait systématiquement à l'efficacité, à l'efficacité dans le déplacement, pour avoir un judo qui se déplace... (Ent 4) ».

Dans toutes ses déclarations, nous percevons chez le professeur DC, ce « déjà-là conceptuel ॥ qui va orienter la transmission des savoirs autour de l'esthétique du geste, de l'efficacité du geste et du plaisir. Mais on ne peut comprendre ce "déjà-là conceptuel » qu'en référence au "déjà-là expérientiel». Celui-ci constitue la clé qui permet de comprendre l'articulation entre les autres "déjà-là » et ce qui va guider les décisions didactiques du professeur DC dans la leçon. L'entretien d'après-coup nous donne des informations complémentaires qui vont nous permettre de comprendre ce qui est à l'origine de ses conceptions mais aussi de ses intentions didactiques. On retrouve dans cet entretien d'après-coup des valeurs attachées à la position, en relation avec la culture japonaise et l'histoire des samouraïs : "Ce qui est général, c'est qu'on se tient droit. Mais pour un judoka, se casser en deux, c'est honteux, c'est rompre. Ce n'est pas de l'orgueil, il faut être droit ; en plus, les samouraïs étaient droits, ils ne devaient pas se casser (AC) ॥. Mais, c'est en connaissant son expérience de judoka que l'on comprend ce qui est à 
l'origine de sa recherche personnelle pour être toujours plus efficace avec ses élèves : "Moi, je suis allé au Japon, j’ai travaillé avec les Japonais. J'ai vu Jean-Luc $X$ qui était beaucoup plus fort que ces judokas japonais. Je l'ai rencontré. Il travaillait droit (AC) ».

La rencontre avec ce judoka exceptionnel, Jean-Luc X, semble déterminante car il incarne un autre judo, un judo fait de beauté et d'efficacité. Les termes employés pour décrire ce judoka exceptionnel, montrent combien le professeur DC a été impressionné par cet homme qui avait le même âge que lui : "II montait s'entraîner à Paris avec l'équipe de France et il les jetait tous (...) X, il m'a fait morote5 debout et je suis passé par-dessus lui ; il le faisait debout, debout, j'étais propulsé ! Alors on me dit, il avait une force phénoménale! (...) ce travail d'X, c'est vraiment quelque chose de phénoménal. J'ai pourtant tiré avec des Japonais qui faisaient morote merveilleusement, qui se glissaient sous moi, mais je n'ai jamais vu un Japonais me faire morote comme lui, je bondissais, on bondissait avec Jean-Luc (AC) ».

Cet homme incarne donc un idéal de beauté et d'efficacité que le professeur DC cherche à transmettre à ses élèves. Cet idéal du geste se traduit par des formes de corps magnifiques, dimension visible de la technique : "Son tai-otoshi, c'était une merveille du genre, c'était beau ; c'était beau ce qu'il faisait. C'était rapide, c'était une forme éblouissante $(A C)$ ». Mais aussi, dimension invisible de la technique, avec des sensations que seul, celui qui combat peut ressentir quand il est tenu par le kumi-kata ${ }^{6}$ : « Jean-Luc, il avait une façon de prendre le kumi-kata, il te rivait dans le sol. Il y a des judokas qui m'ont cassé en deux, ça cela ne m'a jamais impressionné, j'aurais fait deux heures de musculation par jour, je ne me serais jamais laissé casser. Mais $X$, c'était autre chose (AC) ».

Le « déjà-là expérientiel » du professeur DC revêt donc un poids important qui va « filtrer » son enseignement du judo. C'est cette expérience, «incorporée » qui va orienter ses intentions didactiques spécifiques et générales mais aussi son travail de recherche sur le plan didactique : " J'ai toujours essayé de retrouver cela, de le recréer, mais le seul mérite que je me reconnais, c'est de ne pas avoir encore arrêté de rechercher (AC) ").

3. 4. Illustrations avec deux autres études de cas

Dans le cas du professeur JB, nous avons identifié au cours des entretiens quatre points qui caractérisent son « déjà-là intentionnel général » : la sécurité des élèves, le respect de l'adversaire dans le combat, le principe d'action-réaction et le savoir informationnel.

\footnotetext{
${ }^{5}$ Morote-seoi-nage : technique de projection vers l'avant.

${ }^{6}$ Le kumi-kata correspond à la saisie du judogi par l'adversaire.
} 
Concernant le «déjà-là conceptuel » de cet enseignant, nous avons constaté qu'il regroupait une notion importante : «le plaisir du combat». Dans le premier entretien, le professeur JB nous livre certaines intentions didactiques qui semblent relever de ses conceptions : " qu'ils prennent du plaisir à combattre, mais sans se faire mal » (Ent $1 \mathrm{JB})$. C'est le plaisir à travers le plaisir de combattre, mais aussi le plaisir de voir les élèves heureux : " mais ce qui me fait plaisir, c'est d'en voir qui ont le sourire » (Ent 3 JB). II retrouve chez ses élèves un plaisir qu'il a lui-même connu. C'est aussi cela qu'il cherche à transmettre : "II y a toujours moyen de s'amuser avec l'autre pour essayer de le faire tomber. Pour moi, c'est ça le jeu, c'est s'amuser à faire tomber l'autre » (AC JB).

Dans l'entretien d'après-coup lorsque nous avons questionné le professeur JB sur le savoir culturel et plus particulièrement sur l'utilisation des noms japonais, il nous a expliqué pourquoi il mettait volontairement de côté une partie du savoir culturel : « Oui, le côté culturel, c'est le côté gentil ; moi, j'ai quand même côtoyé quelques Japonais sur les tatamis, donc, c'est bon.... (...) Les Japonais, bien sûr, ils ont une culture spécifique, et quand on se trouve sur les tatamis comme cela m'est arrivé quand j'étais très jeune, que l'on se fait complètement massacrer par des gros tas, il ne faut pas exagérer avec le culturel... (AC JB). Progressivement, nous avons pris connaissance de l'histoire personnelle du professeur JB et nous avons ainsi mieux compris pourquoi il opérait une telle sélection dans les savoirs à transmettre en ce qui concernait le savoir culturel : « je trouve qu'il y avait un gros décalage entre ce qu'on me disait des Japonais, et les relations que j'avais avec ces gens-là sur les tatamis. Que ... à chaque fois ils faisaient un combat de championnat, mais il ne fallait pas les faire tomber en randori. Et puis, un gros tas, deux fois plus lourd que toi, il n'hésitait pas à te massacrer, moi je n'avais que quinze ans... ॥ $(A C J B)$.

Ainsi, l'expérience souvent traumatisante du combat avec des judokas japonais amène le professeur JB à occulter volontairement la transmission de certains savoirs culturels. Ce que nous avons appelé le « déjà-là expérientiel » constitue donc bien un filtre important dans la sélection de certains savoirs par l'enseignant, ici, des objets de savoir culturel.

Le cas du professeur GL est lui aussi singulier même s'il présente un « déjà-là conceptuel » qui reprend des éléments que nous avons déjà évoqués chez d'autres enseignants comme l'autonomie et la transmission d'une culture mais il ajoute un point particulier : "ce qui organise mon enseignement, c'est la transmission culturelle, c'est le fait que c'est une activité duelle, un activité assez singulière du fait qu'on est en corps à corps » (ACGL 6). Ce qui nous semble fondamental, dans sa conception, c'est aussi de 
transmettre ce qui fait la spécificité de cette activité physique, c'est-à-dire le corps à corps. II reviendra à plusieurs reprises sur ce point.

Pour ce qui concerne son « déjà-là expérientiel », le professeur GL nous indique pourquoi il choisit d'inclure le judo dans sa programmation d'activité en EPS: "Bon, je suis incapable de dire ce qui se passe dans la tête des gamins, je pense qu'affectivement, il y a quelque chose, il faut aller au combat... C'est quelque chose de particulier dans la vie " $(A C G l)$. Nous avons questionné le professeur Gl sur ce qu'il entendait par «aller au combat ": "II n'y a que les sports de combat duels qui peuvent apporter ce genre d'engagement, qui font travailler ce genre de ressources ; il y a un mélange de technique, de savoir-faire avec son corps pour ne pas se faire mal, pas faire mal à l'autre et inversement, et puis, il y a cette dimension affective importante ... " (AC GL). Nous avons compris finalement que derrière cet engagement physique, derrière ce corps à corps, se cachait un plaisir que l'enseignant nous indique : «Oui, parce que j'ai fait du judo avant, parce que j'ai pris du plaisir à faire du judo avant (de l'enseigner) » (AC GL).

Cette émotion, ce plaisir, qui ne s'acquiert que dans l'engagement corporel, le professeur Gl cherche à le transmettre à ses élèves à tel point qu'il veut éviter tout déplaisir dans la pratique: "j'essaie de voir où ils en sont, quels sont ceux qui peuvent accepter la confrontation directe, c'est le côté sécurité affective qui me tient à cœur, j'y suis très attentif, mais aussi le côté technique, mais je n'aimerais pas mettre l'élève dans la situation de se prendre des coups et qu'il soit mal, c'est ça mon objectif ॥ (AC GL 4).

Plaisir et engagement à fond dans le contact, sans problème affectif, semblent être les éléments fondamentaux qui structurent le « déjà-là expérientiel » du professeur GL. Cet « engagement à fond » est tellement important qu'il le répète à plusieurs reprises lors du second entretien: "L'attaquant risque de ne pas s'engager à fond dans sa première technique. Les blocages qui me font le plus peur, ce sont les blocages de type affectif, les blocages de contact. Dès lors qu'il sait que la riposte est possible, il risque de ne pas s'engager à fond dans sa première attaque » (Ent 2 GL).

À travers ces différentes études du cas des professeurs d'EPS, nous avons montré à quel point l'expérience personnelle pouvait influencer les intentions et les décisions didactiques, qu'elles soient prises avant l'action (les intentions) ou pendant l'action didactique. Cette modélisation des «filtres » personnels ou déterminations personnelles, réalisée à partir de ces cas, a été confirmée chez les quatre professeurs d'EPS ainsi qu'auprès de quatre professeurs de judo (Loizon, 2004b). Dans ce travail scientifique, nous avons essayé de produire « une construction fidèle au fonctionnement du sujet, voire 
de prélever la formule qui rende compte de sa position subjective, afin de transmettre les motifs de son choix » (Terrisse, 2000).

\section{Conclusion}

Notre recherche, centrée initialement sur les savoirs enseignés, nous a effectivement permis d'identifier, différents objets de savoir relatifs au judo qui viennent compléter les savoirs technique et stratégique sur lesquels nous nous étions focalisés au départ de ce travail. Mais elle nous a surtout permis de comprendre, dans les processus interactifs en situation d'enseignement-apprentissage, l'influence des déterminations personnelles, c'est-à-dire comprendre les raisons personnelles qui poussent cet individu particulier à agir comme il le fait. La méthode clinique utilisée, celle du « cas par cas », avec des entretiens post-leçon et des entretiens menés dans l'après-coup, a mis en évidence l'importance de quatre niveaux de « déjà-là» qui filtrent l'action didactique et participent à la détermination des savoirs transmis par les professeurs d'EPS. Ces filtres agissent sur les intentions didactiques, en amont, mais aussi sur les décisions des enseignants, en aval, lorsqu'elles sont prises dans l'urgence des situations d'enseignement.

Nous sommes partis à la recherche des savoirs enseignés, mais au bout du long chemin que constitue ce travail de thèse, nous avons trouvé le sujet, le professeur avec son histoire personnelle. Cette histoire, chaque fois singulière, nous a permis de comprendre dans quelle mesure ces déterminants personnels agissent au sein du système didactique. En ce sens, nous validons ici, pour l'enseignement, les propos de Gauthier: "Aucun individu n'aborde une interaction d'une manière entièrement vierge ; il y prend place, il y joue à partir de ce qu'il connaît déjà, en se servant de son histoire personnelle pour guider son action » (Gauthier, 1997, p. 120).

\section{Bibliographie}

Amade-Escot, C. (2001). De l'usage des théories de l'enseignant. Questions de l'étude des contrats didactiques en éducation physique, dans Le génie didactique, Usages et mésusages des théories de l'enseignement (p. 23-41). Bruxelles, Éditions De Boeck Université.

Astolfi, J.-P. \& Develay, M. (1989). Didactique des Sciences. Paris, PUF, Que sais-je ?

Barbier, J-M. (1996). Savoirs théoriques et savoirs d'action. 2e édition, Paris, PUF.

Bardin, L. (1998). L'analyse de contenu. 9e édition, Paris, PUF. 
Blaquier, J.-L. (2003) L'antiphilosophie de J. Lacan, in, www.Philagora.net/psychanalyse.

Blanchard-Laville, C. (2001). Les enseignants entre plaisir et souffrance. Paris, PUF.

Bru, M. (2002). Pratiques enseignantes: des recherches à conforter et à développer. Revue française de Pédagogie n¹38, 63-74.

Brousseau, G. (1986). Fondements et méthodes en didactique des mathématiques, Recherches en didactique des mathématiques. Grenoble : La Pensée sauvage.

Brousseau, G. (1998). Théorie des situations didactiques. Textes rassemblés et préparés par N. Balacheff, M. Cooper, R. Sutherland, V. Warfield, Recherches en Didactique des Mathématiques. Grenoble : La Pensée sauvage.

Cadière, R., \& Trilles, F. (1998). JUDO, Analyse et propositions pour la pratique de son enseignement. Cahiers des sports, Paris, Revue EPS.

Carnus M.-F. (2001). Analyse didactique du processus décisionnel de l'enseignant d'EPS en gymnastique. Une étude de cas croisés. Thèse de doctorat en sciences de l'éducation, non publiée, université Paul Sabatier, Toulouse III.

Carnus, M.-F. (2003). Analyse didactique du processus décisionnel de l'enseignant d'EPS en gymnastique ; une étude de cas croisés. In C. Amade-Escot (Sous la direction de), Didactique de l'éducation physique, État des recherches (pp. 193-224). Paris, Éditions Revue EPS.

Chapelle, G. (2004). Le Moi, Du normal au pathologique, Auxerre, Éditions Sciences Humaines.

Chevallard, Y. (1991). La transposition didactique. Grenoble, la Pensée Sauvage, nouvelle édition augmentée.

FFJDA (1967). La progression française d'enseignement. Les Cahiers Techniques.

FFJDA (1990). Méthode Française d’Enseignement du judo-jujitsu. Édition FFJDA.

Freud S. (2001) Abrégé de psychanalyse, Paris, PUF, 14e édition.

Gréhaigne, J.-F ., Guillon, R., \& Roche, J. (1993). Les savoirs en sports collectifs à l'école. In G. Bui-Xuan et J. Gleyse (sous la direction de), Enseigner l'éducation physique et sportive (pp. 27-38). Clermont-Ferrand, AFRAPS.

Janicot, D., \& Pouillart, G. (1997). Le Judo, la technique, la tactique, la pratique. Toulouse, éditions Milan.

Johsua, S. (1996). Le concept de transposition didactique n'est-il propre qu'aux mathématiques ? In M. Caillot, C. Raisky, au-delà des didactiques, le didactique (pp. 61-73). Paris, Bruxelles, De Boeck Université. 
Laborde, C., \& Vergnaud, G. (1994). Théorie et concepts fondamentaux. In G. Vergnaud (Coordonné par), Apprentissage et didactique, où en est-on ? (p. 57-93). Paris, Hachette éducation.

Loizon, D. (2000). Modélisation du système d'attaque et de défense en judo. Étude prospective pour la partie spécifique du Brevet d’État d'Éducateur Sportif 3e degré en Judo, Document non publié.

Loizon, D. (2004). Analyse des pratiques d'enseignement du judo : identification du savoir transmis à travers les variables didactiques utilisées par les enseignants en club et en EPS. Thèse de doctorat en sciences de l'éducation, non publiée, université Paul Sabatier, Toulouse III.

Loizon, D. (2005). La transmission des savoirs: le savoir personnel des enseignants, Revue SAVOIRS (à paraître).

Loizon, D., Margnes, É., \& Terrisse, A. (2004). Analyse des pratiques d'enseignement. Les pratiques déclarées par les professeurs de judo, e-Journal (eJRIEPS).5, 63-85.

Loizon, D. \& Terrisse, A. (2002) EPS et compétences déclarées en Combat de Préhension, Communication orale aux 7e JORESCAM, Toulon, 11 et 12 avril 2002,

Margolinas, C. (1993). De l'importance du vrai et du faux dans la classe de mathématiques. Grenoble, La Pensée Sauvage Éditions.

Martinand, J.-L. (1986). Connaître et transformer la matière. Berne, Peter Lang.

Portugais, J. (1998). Esquisse d'un modèle des intentions didactiques, contribution à la didactique des mathématiques. In Brun, Conne, Floris, Schubauer-Léoni (Sous la direction de), Interactions didactiques, Méthodes d'étude du travail de l'enseignant (p. 57-88). Actes des deuxièmes Journées didactiques de La Fouly, Genève, Université de Genève.

Terrisse, A. (1991). Pour une approche dialectique du combat. Revue EPS, 229, 23-26.

Terrisse, A. (1994) La question du savoir dans la didactique des activités physiques et sportives : essai de formalisation, Note de Synthèse pour l'habilitation à diriger les recherches, non publiée, Université Paul Sabatier

Terrisse, A. (2000). Épistémologie de la recherche clinique en sports de combat. In A. Terrisse (Sous la direction de), Recherches en Sports de combat et Arts Martiaux, État des lieux (pp. 95-108), Paris, Éditions revue EPS.

Terrisse, A., \& Léziart, Y. (1997). L'émergence d'une notion : la transposition didactique. Entretiens avec Michel Verret, Les Sciences de l'éducation pour l'ère nouvelle, CERSE, Université de Caen, 30, n³, 5-29. 
Van Der Maren, J.-M. (1996) Méthodes de recherche pour l'éducation, 2e édition, De Boeck Université.

Vergnaud, G. (1994). Apprentissage et didactique, où en est-on ? Paris, Éditions Hachette Éducation.

Vermersch, P. (1994) L'entretien d'explicitation, Paris, ESF éditeur.

Verret, M. (1975). Le temps des études. Paris, Librairie Champion. 